\title{
Thermal infrared imaging reveals that 6-12 month-old babies show different autonomic response to interaction with robot and avatar
}

\author{
by C. Filippini*, D. Cardone*, D. Perpetuini* A.M. Chiarelli*, L. A. Petitto** and A. Merla* \\ * Department of Neurosciences, Imaging and Clinical Sciences, University G. d'Annunzio of Chieti-Pescara, \\ Italy \\ ** NSF Science of Learning Center, Visual Language and Visual Learning, VL2 ; PhD in Educational \\ Neuroscience (PEN) program, Gallaudet University, Washington, DC, USA
}

\begin{abstract}
From birth, infants are immersed in a social environment, often surrounded by artificial intelligent agents (AIAs). However, there is a significant paucity of work on infants' psychophysiological responses, and their related interest, when interacting with AIAs. Here, the psychophysiological responses of infants during interactions with an embodied robot and a virtual human (avatar) presented on a screen are investigated. Understanding infants' psychophysiological/emotional responses to AIAs provides important new knowledge regarding how AIAs impact infants' during the first year of life, which is a period of critical importance for human learning, especially emotional, social, and language learning and higher cognitive growth.
\end{abstract}

\section{Introduction}

Virtual human and robotic agents tend to occupy a growing prevalence in daily life, with applications ranging from entertainment, training, learning, to tutorial systems [1-3]. Anthropomorphic virtual agents can be a powerful technological tool to impact motivational outcomes and facilitate learning, particularly in young children [4-7]. Especially, robots and avatars might play a primary role in this regard $[8,9]$. However, in order to investigate the quality of the interaction between infants and artificial intelligent agents (AIAs), it is fundamental to monitor the user's psychophysiological state [10]. To this end, contactless technologies that are able to detect psychophysiological states and preserve the ecological features of the infants-AIAs interaction are preferable. By contrast, classical technologies used for monitoring physiological parameters and autonomic nervous system (ANS) activity (e.g. Heart Rate Variability, Skin Conductance Level) typically require contact sensors, which cause manipulation of the infants' body, potentially biasing the measurement of psychophysiological/emotional states.

Advances in thermal infrared (IR) imaging technologies allow monitoring human autonomic functions and inferring psychological and affective states in a contactless manner [11-16] and have proven highly suitable to investigate humanAIAs interaction [5]. Thermal IR imaging is a non-invasive technique that allows measuring the spatial and temporal distribution of the body's surface temperature $[13,17,18]$. The modulation of the cutaneous temperature is related to the vasodilatation and vasoconstriction regulated by the autonomic nervous system (ANS) which is also responsible for the human body homeostasis and the physiological responses to emotional stimuli $[10,19]$.

Thermal data analysis is mainly based on linear descriptive metrics (e.g. average temperature variation from a baseline) indicative of the autonomic activation. More recently, non-linear analysis methods in both frequency and time domain have been applied to IR imaging in order to investigate the autonomic functions [20,21].

So far, thermal IR imaging has been employed for measuring facial skin temperature in infants. For instance, Mizukami et al. used thermal IR imaging as an index of mental stress in 8-to15-weeks-old infants when they were separated from their mothers [22]. Nakanishi and Matsumura investigated changes in facial skin temperature in 2 to 8-months-old infants, when they were laughing, as typical behavior of pleasant and joyful emotion [23]. Aureli et al. assessed the facial temperature variation in 3- to 4- months-old infants, in order to explore the natural human process of attachment between baby and mother, and then the effects of non-responsiveness on the part of the mother [24].

In the present study, we conducted an original investigation of the psychophysiological correlates of autonomic nervous system responses that occurred when infants were interacting with a robot as compared to a virtual human/avatar. The data were drawn from a larger innovative project to create an effective natural language learning tool for young infants, be they hearing or deaf, during the critical periods of human learning and development, called RAVE (or, Robot, $\underline{\text { AVatar, }}$ thermal Enhanced language learning tool) [5-7]). However, entirely new scientific questions and hypotheses are here addressed, and novel analyses that render original scientific findings are conducted. As social and environmental engagement are essential elements in infant learning [Petitto et al. [25]], RAVE used a physically-present (embodied) robot and a virtual human on a TV screen, with the virtual human providing engaging social gestures and linguistic interactions such as linguistic nursery rhymes (NR) in American sign language (ASL). Scientific questions remain concerning whether the two artificial agents elicit different or similar psychophysiological responses in humans. This is because changes in psychophysiological responses are indicative of differences in emotional, social, and attentional engagement. Answering this question with Thermal IR Imaging offers an important scientific advance in our ability to evaluate an AIA's capacity to 
effect learning in young infants, as traditional means of studying the nature of engagement in infants can be inconclusive (e.g., with baby gaze).

In this study, a first-time thermal infrared imaging-based technology (with novel integration of face-tracking and computational modeling) was created specifically to investigate infants' psychophysiological responses [26]. The dynamics of the Thermal IR facial cutaneous temperature was recorded without any contact with the infant participant, thereby making it possible to assess ANS activity and to infer the infant participants' psychophysiological states with a greater ecological validity [27]. The main scientific question was as follows: Does the baby's interaction with the two different AIAs (robot, avatar) evoke different or similar baby psychophysiological responses?

\section{Materials and Methods}

\subsection{Participants}

Sixteen hearing infants (ages 6-12 months) participated in this study. Parents were provided with a detailed explanation of the study's procedures, questions were encouraged, informed consent was invited and obtained. The measurements were performed in agreement with the ethical standards of the Helsinki Declaration [28] and approved by the Institutional Review Board of Gallaudet University, Washington, D.C.. Participation was strictly voluntary, and parents were informed that they could withdraw at any time without consequence or penalty. Demographic information, such as age and gender, were gathered from all the participants. All data were anonymized with participant number IDs to preserve confidentiality and then entered into a database.

\subsection{Experimental paradigm}

Infants were positioned on their parents' laps, comfortably seated on a non-swiveling chair. The experimental design consisted of an initial period of familiarization [5-7,29]. Here, babies could interact with the environment and the experimental setup. Following familiarization, the study design consisted of two (2) conditions. C1: Robot's (RO) interaction with the baby, ending with the robot turning its head towards the TV screen (which contained the avatar) and C2: Avatar's (AV) interaction with the baby. The robot's interactive behavior towards the baby consisted of performing a series of specific head and eye movements; these included, socially communicative gesture ("yes" head nod), "surprise" gesture (head nod forward/backward, eyes wide open+blink), "peek-a-boo" gestures (eyes fully opened and eyes fully closed, plus raised/lowered head nods, as if playing "peek-a-boo"), directed eye gaze (e.g. towards baby, towards TV screen), and waking-up gesture (head bob, plus eye blinks, as if waking up). The avatar's interactive behavior towards the baby consisted of performing different types of hand movements that included socially communicative gestures (e.g., waving $\mathrm{HI}$, BYE), nonaction behavioral gestures (idle pose, as if thinking), and fully linguistic productions such as Nursery Rhymes (NR) in American Sign Language, with grammatically correct linguistic sentences. Further, the ASL Nursery Rhymes had linguistic rhyme structure [Petitto et al. [30]] and age-appropriate semantic content [Petitto et al [25]]. Each condition lasted 8 seconds, with 6 repetitions. To ensure that one condition was not repeated twice in a row, which would thus result in loss of baby's attention, conditions were not randomized. The type of productions that the robot and the avatar could perform are reported in Table 1. Figure 1a shows the ecological experimental environment and setup, with the robot, the avatar on the TV screen, and the baby sitting on his father's lap.

Table 1. List of production type performed by the robot and the avatar

\begin{tabular}{|c|c|c|}
\hline Category (Type) of Productions & $\begin{array}{c}\text { Physically Present } \\
\text { Robot }\end{array}$ & $\begin{array}{c}\text { Virtual human, Avatar } \\
\text { on TV screen }\end{array}$ \\
\hline Behavioural gestures & \\
\hline Communicative/ social gestures & & \\
\hline Linguistic gestures & & \\
\hline
\end{tabular}

\subsection{Thermal IR imaging data processing}

\subsubsection{Data acquisition}


The subject's psychophysiological response was inferred through his/her facial cutaneous temperature changes over time, recorded by thermal camera FLIR A655sc. The camera was equipped with a Focal Plane Array of $640 \times 480$ detectors, 0.02-s time resolution, $0.03 \mathrm{~K}$ temperature sensitivity/Noise Equivalent Temperature Difference, and the capability of collecting the thermal radiation in the $7.5-14 \mu \mathrm{m}$ band. The thermal camera response was blackbody-calibrated in order to remove noise-effects related to the sensor drift/shift dynamics and optical artifacts [13,20]. The sampling rate for thermal imaging was set at 10 frames/s. All the observations were made in a climate-controlled room according to the International Academy of Thermology (IACT) guidelines [31]. The room temperature was set at $23 \pm 1^{\circ} \mathrm{C}$; relative humidity at $50-55 \%$ with no direct ventilation on the subject and no direct sunlight. The observations were located in a dedicated laboratory and took place at approximately the same time of the day [32]. The thermal camera was focused on the infant's face.

\subsubsection{Data analysis}

A visual inspection of the changes in facial thermal imprints in all subjects was performed to qualitatively investigate the infant's autonomic responses and to check the quality of the recording (e.g. exaggerated motion of the participant) throughout the experiment [33]. This analysis was followed by a quantitative estimation of temperature variations of Regions of Interest (ROIs), located in the infant's face (Figure 1c). In particular, to monitor the modulation of the autonomic activity, the nasal tip (NT) area and the supraorbital area associated with the activity of the corrugator muscle (corrugator region (CR)), were selected according to previous studies with infants populations [23,34]. Indeed, these two regions have been shown to be highly sensitive to the psychophysiological activity [15,35-38]. Because the infant was allowed to move its head without any restriction, a soft-tissue tracking algorithm was used to track the NT and CR areas throughout all the images of the time-series. The algorithm allowed to correct the ROls positioning upon movements of the subject's head and accurately compute the ROIs temperature from each facial thermogram. The tracking algorithm, based on a facial landmark detection over time, was developed in Matlab and employed by Manini et al. [33]. It relayed on the evaluation of the 2-D cross-correlation between a target template, chosen by the user in the initial frame of the thermal video, and a similar ROI in a larger searching area, expected to contain the desired template in each of the following frames [39]. In this way, it was possible to automatically extract the thermal signals in the defined regions of interests during the whole experiment. The thermal signals extracted from the 2 ROls, were further corrected from residual motion artifacts. Motion errors were identified and replaced using a linear interpolation of the value neighboring the artifact. An example of an infant's NT and CR temperature time course after being corrected for motion artifact is shown in Figure 1b

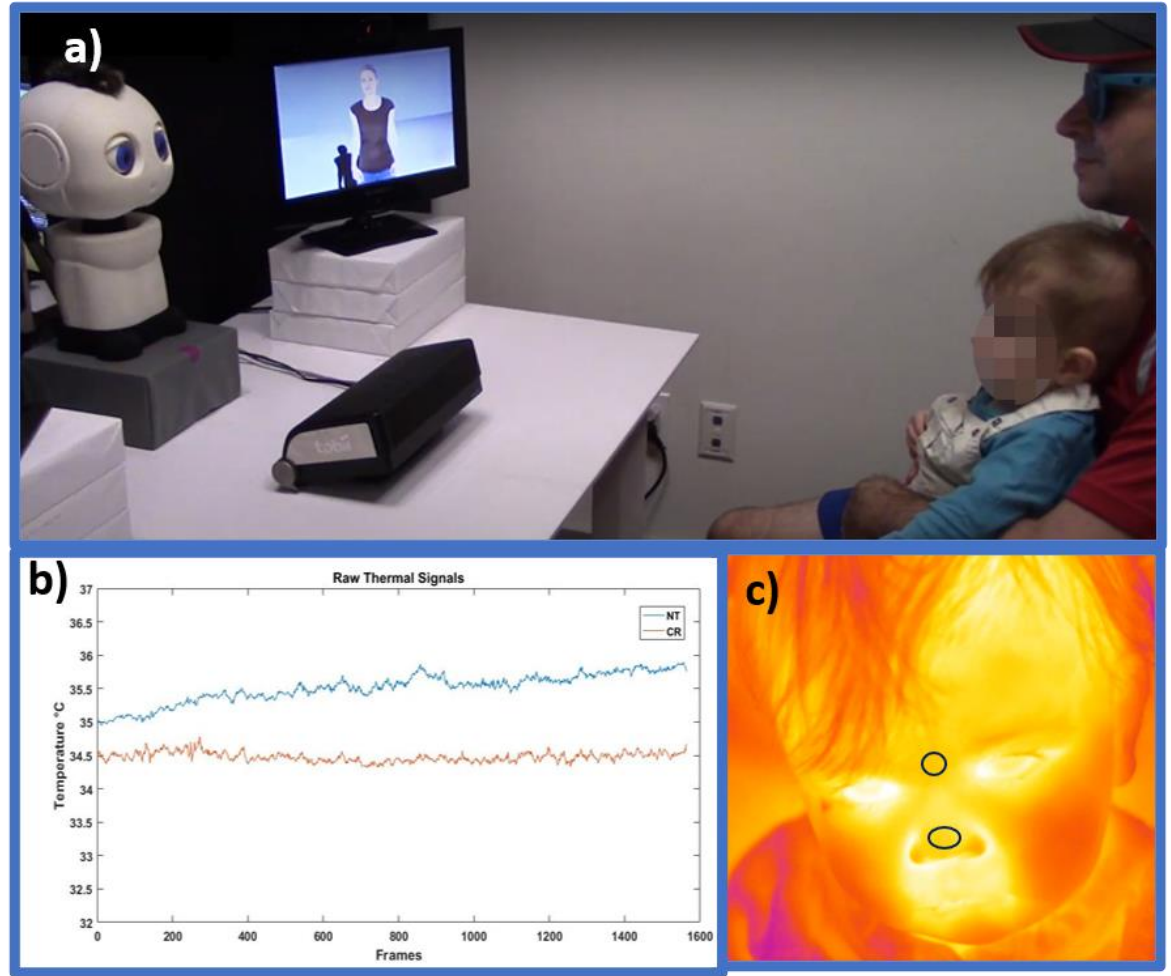

Fig.1 (a) Temperature time course within the ROls extracted through the tracking algorithm and corrected for motion artifacts for further analysis. (b) Example of selected ROIs (blue circle on baby's NT and CR) of a representative baby overlaid onto the baby's first frame of thermal video recording. (c) Experimental environment and setup with the robot the avatar on the TV screen and the baby sitting on his father's lap. 
Thermal data were analyzed employing both a time and a time-frequency domain approach. For the first analysis, the thermal signals were filtered with a bidirectional third-order Butterworth low-pass $2 \mathrm{~Hz}$ filter in order to reduce highfrequency noise. The temperature time course was then normalized and averaged over each condition for each participant. The average normalized temperature during $\mathrm{RO}$ and $\mathrm{AV}$ was further evaluated with respect to the familiarization period by subtracting the average normalized temperature value of the familiarization period from each condition [20].

Concerning the second approach, in order to analyze the thermal signals at different frequencies, but without loss of timing information, a Wavelet transform was performed on both signals. In fact, thermal signals are nonstationary in nature and thermal response time in infants can be different from subject to subject. The frequency bandwidth preserved by the filter included bands in which the skin blood flow oscillations occur, corresponding to the endothelium-related metabolic $(0.008-0.02 \mathrm{~Hz})$, neurogenic $(0.02-0.05 \mathrm{~Hz})$, myogenic $(0.05-0.15 \mathrm{~Hz})$, respiratory $(0.15-0.4 \mathrm{~Hz})$ and cardiac regulations $(0.4-2 \mathrm{~Hz})$ [40]. Exploring these frequency bands allowed to investigate the principal factor that contributed to the signal frequency content in the different experimental conditions. A continuous wavelet transform (Morse wavelet, gamma equal to 3 and time-bandwidth product equal to 60) was performed to investigate the frequency at which the highest signal power occurred $\left(f_{H P}\right)$. The wavelet transform provides a multiresolution analysis by decomposing the signals into a time-frequency space. The signal power content can be thus evaluated as a function of frequency and time [41].

Finally, a coherence analysis between the 2 ROls signal was conducted to investigate the presence of common oscillatory behavior and identify the frequency at which the highest coherence occurred $\left(f_{\mathrm{HC}}\right)$. The analysis was performed using a wavelet phase coherence approach [42], the coherence was computed using the analytic Morlet wavelet. As with other types of transforms, the wavelet coherence applied to a finite length time-series inevitably suffers from border distortions. The region in which the transform suffers from these edge effects is called the cone of influence (COI). Similarly, to spectral analysis, errors will occur at the beginning and ends of the spectrum because of the limited time series. Therefore, the familiarization period and the sixth conditions' repetition, which were not included in the COI, were disregarded from $\mathrm{fHP}_{H P}$ and $\mathrm{f}_{\mathrm{HC}}$ further analysis. Wavelet coherence values as a function of frequency and time of a representative infant is reported in Figure 2. Transparent regions outside the black line (COI) delineate regions where edge effects are significant.

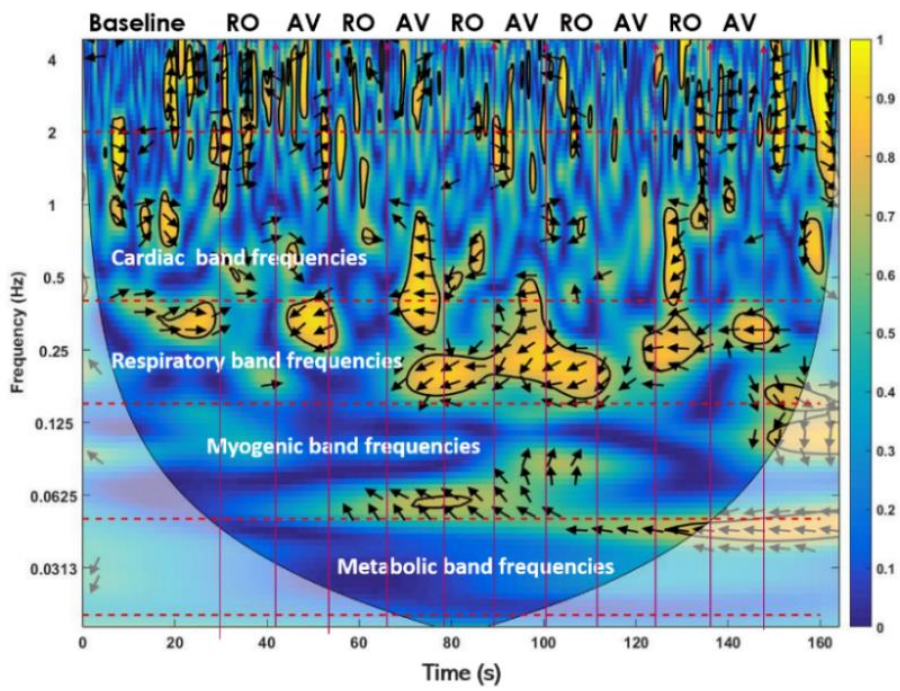

Fig. 2: Wavelet coherence between NT and CR thermal signals of a representative subject in both time and frequency domain. The black contours surround the 95\% statistical significance area against red noise background assessed by Monte Carlo methods. In areas where the coherence exceeds 0.5, arrows are displayed to show the phase lag of CR with respect to NT thermal signal.

\subsection{Statistical analysis}

Statistical analyses were performed using SPSS software (IBM SPSS Statistics 19). The One-way repeated measure ANOVA was computed on the conditions' (RO and AV) average temperature of both ROls. Furthermore, it was performed on the conditions' average value of $f_{H P}$, and $f_{H C}$. The $p$-values were corrected for multiple comparisons using the Bonferroni adjustment. The goal of this analysis was to infer the autonomic response of the subjects and answer the theoretical question underlying the present study i.e. whether or not the baby's interaction with the two different AIAs evoke different baby psychophysiological responses. 


\section{Results}

Regarding the time domain analysis, the one-way analysis of variance showed a significant difference in average temperature on NT between the 2 conditions $(F(1,15)=4.94, p=0.04)$ but not for CR. The results revealed a decrease in average temperature during the avatar/AV condition with respect to the robot/RO condition.

Concerning the time-frequency analysis, both NT and CR signals showed a statistically significant difference in the $f_{H P}$ during $A V$ and $R O(N T: F(1,15)=7.29, p=0.02$; $C R: F(1,15)=9.49, p=0.01)$. AV exhibited a stronger respiratory activity since the AV $f_{H P}$ found on the respiratory band was statistically higher with respect to the RO $f_{H P}$. This result suggests a higher breathing rate during the AV compared to RO condition. This evidence was also supported by the coherence analysis results, indicating a frequency coherence peak on the respiratory band during AV condition and on the cardiac band during the $R O(F(1,15)=9.07, p=0.01)$. The statistical results are summarized in Table 2 .

Table 2. Results of the test for differences between the 2 conditions (RO and AV). The significance has been tested trough a one-way repeated measure ANOVA.

\begin{tabular}{|l|l|l|l|}
\hline & $\begin{array}{c}\text { RO } \\
\text { (mean value } \pm \\
\text { standard error) }\end{array}$ & $\begin{array}{c}\text { AV } \\
\text { (mean value } \pm \\
\text { standard error) }\end{array}$ & \multicolumn{1}{|c|}{ Significance } \\
\hline NT Average Temperature & $0,53 \pm 0,02$ & $0,47 \pm 0,03$ & $F(1,15)=4,94 ; p=0,04$ \\
\hline CR Average Temperature & $0,52 \pm 0,03$ & $0,51 \pm 0,03$ & $F(1,15)=0,032 ;$ n.s. \\
\hline NT f $f_{H P}$ & $0,18 \pm 0,03 \mathrm{~Hz}$ & $0,28 \pm 0,05 \mathrm{~Hz}$ & $F(1,15)=7,29 ; p=0,02$ \\
\hline CR fHP & $0,23 \pm 0,05 \mathrm{~Hz}$ & $0,31 \pm 0,05 \mathrm{~Hz}$ & $F(1,15)=9,49 ; p=0,01$ \\
\hline NT-CR $f_{H C}$ & $0,53 \pm 0,03 \mathrm{~Hz}$ & $0,44 \pm 0,03 \mathrm{~Hz}$ & $F(1,15)=9,07 ; p=0,01$ \\
\hline
\end{tabular}

\section{Discussion}

In recent years, artificial intelligent agents have received increasing attention because of their use in smart applications that affect multiple aspects of daily life. Mostly research involving children (and to a lesser extent young infants) has focused on robots that provide social and cognitive support, the so-called "socially assistive robots." Here, children have shown their understanding of humanoid robots as being socially communicative agents [43,44]. Conversely, lesser attention has focused on virtual humans/avatars that are represented on a screen, such as a TV screen/monitor and other contemporary smart devices (e.g., iPads, smartphones) despite the fact that this usage is now ubiquitous. In fact, among the small number of studies that do exist, researchers have argued that placing young infants in front of a TV screen is judged to be an ineffectual practice [45], [46].

In the present study, we conducted a novel investigation of the psychophysiological responses of young babies during interactions with a physically present/embodied robot versus a virtual human/avatar presented on a TV screen. The overarching goal was to shed new light on the powerful contribution that advances in Thermal IR Imaging have as an innovative tool to make possible new answers about the nature of infant social, emotional, and higher cognitive development, as well as early learning, hitherto not possible with traditional behavioral methods. Here, our use of Thermal IR Imaging - a non-invasive and contactless technology-permitted an ecological testing environment where babies were free to move and rest on a parent's lap without any major restrictions. As a further advance, we created an entirely novel infant perceptual measurement system that was built by computationally integrating thermal IR imaging with affective facetracking.

Using such innovations in Thermal IR Imaging, we asked whether babies would show the same or different psychophysiological responses to the robot versus the avatar. Because the robot was physically present and produced socially-communicative head and eye-contact gestures directly to the babies-as compared to the virtual human/avatar on a TV screen, we hypothesized that the babies may show robust psychophysiological markers of attention and engagement largely to the socially potent robot (-perhaps even ignoring the flat TV screen with the avatar). 
Our Thermal IR results revealed the surprising: Babies showed powerful psychophysical responses (indicative of Autonomic Nervous System regulation) both to the robot and to the avatar. Most interestingly, the Thermal IR evidence demonstrated that the babies' psychophysical responses were different for each Artificial Agent. An increase in nose tip temperature was observed when babies interacted with the robot, suggesting more social engagement. This finding corroborates earlier studies in child development showing that older toddlers and children can perceive robots as socially communicative [43,44], although no other team has investigated this question in infants as young as studied here (ages 612 months).

By contrast, a drop in nose tip temperature was observed when babies interacted with the avatar. This occurred in combination with a higher $f_{H P}$ in the respiratory band not seen in the robot condition. These intriguing findings indicate that the babies were in a heightened state of attention-vigilance engagement when interacting with the avatar, as if the avatar was eliciting sustained scrutiny and tacit analysis. That a drop in nose tip temperature is associated with increased attentional engagement corroborates our earlier Thermal IR Imaging studies of adults [17, 44], and the increased respiratory response found during heightened attention as observed in adults [45]. As one novel hypothesis for future study, we suggest that the drop in nose tip temperature and increase in respiratory response-measurements made uniquely possible with Thermal IR Imaging-may serve as an innovative psychophysiological marker, not only to indicate a baby's heightened attentional state, but, correspondingly, of being cognitively poised or "Ready to Learn." In turn, this psychophysiological marker of a baby's state of being "Ready to Learn" may one day have broad transformative translational impact in the development of learning and teaching smart apps for use with young babies.

One intriguing question resulting from the present findings is why? Why did babies show heightened attention to the avatar? Why was this so even though the avatar was on a flat TV screen (as opposed to the physically present robot) and even though these hearing (non sign-exposed) babies, by design, were selected for participation in the study because they would not understand the meanings of the avatar's predominant productions-specifically, the production of fully linguistic nursery rhymes in American Sign Language? While this is a question for future analysis and experiments, our previous findings with infants provide insight. Because human babies exhibit peaked attention to the maximally contrasting, rhythmic temporal patterning of spoken language during the critical period of 6-12 months, we hypothesize that these present babies' attention/fascination with the avatar was due to the avatar's production of the perceptually-salient (but meaningless) patterns of language being produced by the avatar, and more: The babies were attending to the language patterns, and most remarkably here, attending even though the language patterns were produced on the hands in American Sign Language as opposed to being produced on the tongue in spoken language. Surprisingly, the findings suggest that this stage of human language acquisition occurs universally across all world languages, be they signed languages or spoken languages (see especially Petitto et al. [47]).

Future studies are needed to investigate further a wider range of psychophysiological states as they are associated with corresponding emotional, social, and attentional responses in babies when interacting with different types of AlAs. This is needed especially in light of the intriguing implications raised here regarding the potential role of artificial avatar agents to facilitate learning in human infants. Further study is needed to assess how the specific content of the artificial agent's communicative exchanges contribute to the different psychophysiological responses that were observed (for example, both the avatar's linguistic nursery rhymes, social gestures, and neutral behaviors and the robot's different communicative exchanges). It would also be important to increase the sample size to include deaf infants, even though it must be noted that it is difficult and highly complex to recruit profoundly deaf infants (and families willing to participate in research) as their incidence is very low in the general population. Moreover, a valuable contribution to science involves the integration of Thermal IR Imaging with other neuroimaging technologies, such as functional near-infrared spectroscopy (fNIRS) brain imaging. Presently, we are using this thermal-fNIRS brain imaging integrated technology to gain even greater insight into the cognitive, emotional, and social factors underlying learning and higher cognitive development, and how these factors relate to maturational stages of brain growth in very young babies.

\section{Conclusion}

The present results provide compelling evidence of how the use of Thermal Infrared Imaging can be a powerful tool to investigate human-artificial agent interactions, even when studying challenging populations, such as young babies ages 6 to 12 months old. The study revealed for the first time insights into infants' psychophysiological responses to intelligent artificial agents, and, remarkably, the subtypes of responses that each of these agents elicited, be they engagement that is more socially based (as seen in the babies' interactions with the robot) versus engagement involving more sustained attention and vigilance (as seen in the babies' the avatar). This work paves the way for a novel and smart procedure to gain vital psychophysiological inference during infants-AIAs interactions that have the potential to render revolutionary advance across multiple applications, such as research, and, crucially, assistive application platforms and frameworks to advance human learning in infants and across the lifespan. Especially exciting, understanding infants' psychophysiological states as associated with emotional, social, and cognitive responses to artificial intelligent agents may provide important new knowledge regarding how they can facilitate learning and higher cognitive, social, and emotional growth in young infants during the first year of life, and beyond. 
Funding: This work was supported by the W.M. Keck Foundation (PI: Petitto), National Science Foundation (IIS-1547178, PI: Petitto). PON FESR MIUR R\&I 2014-2020 - Asse II - ADAS+ (ARS01_00459, PI: Merla) and PON MIUR SI-ROBOTICS (ARS01_01120, PI: Merla).

\section{REFERENCES}

1. Scheutz, M. Useful Roles of Emotions in Artificial Agents: A Case Study from Artificial Life. 6.

2. Mubin, O.; Stevens, C.J.; Shahid, S.; Al Mahmud, A.; Dong, J.-J. A review of the applicability of robots in education. J. Technol. Educ. Learn. 2013, 1, 13.

3. Belpaeme, T.; Baxter, P.; Read, R.; Wood, R.; Cuayáhuitl, H.; Kiefer, B.; Racioppa, S.; Kruijff-Korbayová, I.; Athanasopoulos, G.; Enescu, V. Multimodal child-robot interaction: Building social bonds. J. Hum.-Robot Interact. 2013, 1, 33-53.

4. Baylor, A.L. Promoting motivation with virtual agents and avatars: role of visual presence and appearance. Philos. Trans. R. Soc. B Biol. Sci. 2009, 364, 3559-3565.

5. Scassellati, B.; Brawer, J.; Tsui, K.; Nasihati Gilani, S.; Malzkuhn, M.; Manini, B.; Stone, A.; Kartheiser, G.; Merla, A.; Shapiro, A.; et al. Teaching Language to Deaf Infants with a Robot and a Virtual Human. In Proceedings of the Proceedings of the $2018 \mathrm{CHI}$ Conference on Human Factors in Computing Systems; ACM: New York, NY, USA, 2018; pp. 553:1-553:13.

6. Nasihati Gilani, S.; Traum, D.; Sortino, R.; Gallagher, G.; Aaron-lozano, K.; Padilla, C.; Shapiro, A.; Lamberton, J.; Petitto, L. Can a Virtual Human Facilitate Language Learning in a Young Baby? In Proceedings of the Proceedings of the 18th International Conference on Autonomous Agents and MultiAgent Systems; International Foundation for Autonomous Agents and Multiagent Systems: Richland, SC, 2019; pp. 2135-2137.

7. Nasihati Gilani, S.; Traum, D.; Sortino, R.; Gallagher, G.; Aaron-Lozano, K.; Padilla, C.; Shapiro, A.; Lamberton, J.; Petitto, L.-A. Can a Signing Virtual Human Engage a Baby's Attention? In Proceedings of the Proceedings of the 19th ACM International Conference on Intelligent Virtual Agents; ACM: New York, NY, USA, 2019; pp. $162-169$.

8. Rickel, J. Intelligent virtual agents for education and training: Opportunities and challenges. In Proceedings of the International Workshop on Intelligent Virtual Agents; Springer, 2001; pp. 15-22.

9. Fitter, N.T.; Funke, R.; Pulido, J.C.; Eisenman, L.E.; Deng, W.; Rosales, M.R.; Bradley, N.S.; Sargent, B.; Smith, B.A.; Mataric, M.J. Socially assistive infant-robot interaction: Using robots to encourage infant leg-motion training. IEEE Robot. Autom. Mag. 2019, 26, 12-23.

10. Filippini, C.; Perpetuini, D.; Cardone, D.; Chiarelli, A.M.; Merla, A. Thermal Infrared Imaging-Based Affective Computing and Its Application to Facilitate Human Robot Interaction: A Review. Appl. Sci. 2020, $10,2924$.

11. Engert, V.; Merla, A.; Grant, J.A.; Cardone, D.; Tusche, A.; Singer, T. Exploring the Use of Thermal Infrared Imaging in Human Stress Research. PLoS ONE 2014, 9, doi:10.1371/journal.pone.0090782.

12. Cardone, D.; Pinti, P.; Merla, A. Thermal Infrared Imaging-Based Computational Psychophysiology for Psychometrics Available online: https://www.hindawi.com/journals/cmmm/2015/984353/abs/ (accessed on Jan 4, 2020).

13. Cardone, D.; Merla, A. New frontiers for applications of thermal infrared imaging devices: Computational psychopshysiology in the neurosciences. Sensors 2017, 17, 1042.

14. Panasiti, M.S.; Cardone, D.; Pavone, E.F.; Mancini, A.; Merla, A.; Aglioti, S.M. Thermal signatures of voluntary deception in ecological conditions. Sci. Rep. 2016, 6, 1-10.

15. Merla, A.; Romani, G.L. Thermal Signatures of Emotional Arousal: A Functional Infrared Imaging Study. In Proceedings of the 2007 29th Annual International Conference of the IEEE Engineering in Medicine and Biology Society; 2007; pp. 247-249.

16. Merla, A. Revealing Psychophysiology and Emotions through Thermal Infrared Imaging. In Proceedings of the PhyCS; 2014; pp. 368-377.

17. Perpetuini, D.; Cardone, D.; Bucco, R.; Zito, M.; Merla, A. Assessment of the Autonomic Response in Alzheimer's Patients During the Execution of Memory Tasks: A Functional Thermal Imaging Study. Curr. Alzheimer Res. 2018, 15, 951-958.

18. Filippini, C.; Spadolini, E.; Cardone, D.; Merla, A. Thermal Imaging Based Affective Computing for Educational Robot. Proceedings 2019, 27, 27, doi:10.3390/proceedings2019027027.

19. Ebisch, S.J.; Aureli, T.; Bafunno, D.; Cardone, D.; Romani, G.L.; Merla, A. Mother and child in synchrony: thermal facial imprints of autonomic contagion. Biol. Psychol. 2012, 89, 123-129.

20. Pinti, P.; Cardone, D.; Merla, A. Simultaneous fNIRS and thermal infrared imaging during cognitive task reveal autonomic correlates of prefrontal cortex activity. Sci. Rep. 2015, 5, 1-14, doi:10.1038/srep17471.

21. Perpetuini, D.; Cardone, D.; Chiarelli, A.M.; Filippini, C.; Croce, P.; Zappasodi, F.; Rotunno, L.; Anzoletti, N.; Zito, M.; Merla, A. Autonomic impairment in Alzheimer's disease is revealed by complexity analysis of functional thermal imaging signals during cognitive tasks. Physiol. Meas. 2019, 40, 034002.

22. Mizukami, K.; Kobayashi, N.; Ishii, T.; Iwata, H. First selective attachment begins in early infancy: A study using telethermography. Infant Behav. Dev. 1990, 13, 257-271, doi:10.1016/0163-6383(90)90034-6. 
23. Nakanishi, R.; Imai-Matsumura, K. Facial skin temperature decreases in infants with joyful expression. Infant Behav. Dev. 2008, 31, 137-144, doi:10.1016/j.infbeh.2007.09.001.

24. Aureli, T.; Grazia, A.; Cardone, D.; Merla, A. Behavioral and facial thermal variations in 3-to 4-month-old infants during the Still-Face Paradigm. Front. Psychol. 2015, 6, doi:10.3389/fpsyg.2015.01586.

25. Petitto, L.A.; Langdon, C.; Stone, A.; Andriola, D.; Kartheiser, G.; Cochran, C. Visual sign phonology: insights into human reading and language from a natural soundless phonology. WIREs Cogn. Sci. 2016, 7, 366-381, doi:10.1002/wcs.1404.

26. Merla, A. Thermal expression of intersubjectivity offers new possibilities to human-machine and technologically mediated interactions. Front. Psychol. 2014, 5, doi:10.3389/fpsyg.2014.00802.

27. Ioannou, S.; Ebisch, S.; Aureli, T.; Bafunno, D.; loannides, H.A.; Cardone, D.; Manini, B.; Romani, G.L.; Gallese, V.; Merla, A. The Autonomic Signature of Guilt in Children: A Thermal Infrared Imaging Study. PLOS ONE 2013, 8, e79440, doi:10.1371/journal.pone.0079440.

28. Helsinki, D. of Recommendations guiding physicians in biomedical research involving human subjects. JAMA 1997, 277, 925-926.

29. Meltzoff, A.N.; Brooks, R. Self-experience as a mechanism for learning about others: a training study in social cognition. Dev. Psychol. 2008, 44, 1257.

30. Petitto, L.A.; Holowka, S.; Sergio, L.E.; Ostry, D. Language rhythms in baby hand movements. Nature 2001, 413, 35-36, doi:10.1038/35092613.

31. Thermology, I.A. of C. Thermography guidelines: standards and protocols in clinical thermographic imaging; IACT, 2002;

32. Marins, J.C.B.; Formenti, D.; Costa, C.M.A.; de Andrade Fernandes, A.; Sillero-Quintana, M. Circadian and gender differences in skin temperature in militaries by thermography. Infrared Phys. Technol. 2015, 71, 322-328.

33. Manini, B.; Cardone, D.; Ebisch, S.; Bafunno, D.; Aureli, T.; Merla, A. Mom feels what her child feels: thermal signatures of vicarious autonomic response while watching children in a stressful situation. Front. Hum. Neurosci. 2013, 7, doi:10.3389/fnhum.2013.00299.

34. Aureli, T.; Grazia, A.; Cardone, D.; Merla, A. Behavioral and facial thermal variations in 3-to 4-month-old infants during the Still-Face Paradigm. Front. Psychol. 2015, 6, doi:10.3389/fpsyg.2015.01586.

35. Zhu, Z.; Tsiamyrtzis, P.; Pavlidis, I. Forehead thermal signature extraction in lie detection. In Proceedings of the 2007 29th Annual International Conference of the IEEE Engineering in Medicine and Biology Society; IEEE, 2007; pp. 243-246.

36. Puri, C.; Olson, L.; Pavlidis, I.; Levine, J.; Starren, J. StressCam: non-contact measurement of users' emotional states through thermal imaging. In Proceedings of the $\mathrm{CHI}$ '05 extended abstracts on Human factors in computing systems; 2005; pp. 1725-1728.

37. Shastri, D.; Merla, A.; Tsiamyrtzis, P.; Pavlidis, I. Imaging facial signs of neurophysiological responses. IEEE Trans. Biomed. Eng. 2008, 56, 477-484.

38. Di Giacinto, A.; Brunetti, M.; Sepede, G.; Ferretti, A.; Merla, A. Thermal signature of fear conditioning in mild post traumatic stress disorder. Neuroscience 2014, 266, 216-223.

39. Cardone, D.; Merla, A. The thermal dimension of psychophysiological and emotional responses revealed by thermal infrared imaging. In Proceedings of the 2014 IEEE International Conference on Image Processing (ICIP); IEEE, 2014; pp. 1942-1946.

40. Podtaev, S.; Morozov, M.; Frick, P. Wavelet-based Correlations of Skin Temperature and Blood Flow Oscillations. Cardiovasc. Eng. 2008, 8, 185-189, doi:10.1007/s10558-008-9055-y.

41. Grinsted, A.; Moore, J.C.; Jevrejeva, S. Application of the cross wavelet transform and wavelet coherence to geophysical time series. Nonlinear Process. Geophys. 2004, 11, 561-566.

42. Grinsted, A. grinsted/wavelet-coherence; 2020;

43. Arita, A.; Hiraki, K.; Kanda, T.; Ishiguro, H. Can we talk to robots? Ten-month-old infants expected interactive humanoid robots to be talked to by persons. Cognition 2005, 95, B49-B57, doi:10.1016/j.cognition.2004.08.001.

44. Meltzoff, A.N.; Brooks, R.; Shon, A.P.; Rao, R.P. "Social" robots are psychological agents for infants: A test of gaze following. Neural Netw. 2010, 23, 966-972.

45. Anderson, D.R.; Pempek, T.A. Television and Very Young Children. Am. Behav. Sci. 2005, 48, 505-522, doi:10.1177/0002764204271506.

46. Kuhl, P.K.; Tsao, F.-M.; Liu, H.-M. Foreign-language experience in infancy: Effects of short-term exposure and social interaction on phonetic learning. Proc. Natl. Acad. Sci. 2003, 100, 9096-9101, doi:10.1073/pnas.1532872100.

47. Petitto, L.A.; Berens, M.S.; Kovelman, I.; Dubins, M.H.; Jasinska, K.; Shalinsky, M. The "Perceptual Wedge Hypothesis" as the basis for bilingual babies' phonetic processing advantage: New insights from fNIRS brain imaging. Brain Lang. 2012, 121, 130-143, doi:10.1016/j.bandl.2011.05.003. 